\title{
A study on cutaneous adverse drug reactions at district Mc. Gann teaching hospital, Shimoga institute of medical sciences, Shivamogga, Karnataka, India
}

\author{
Jean Lourdes Murray ${ }^{1}$, Vedavathi $\mathbf{H}^{1}$, Nagaraja S. Prasad ${ }^{1}$, Dadapeer $\mathbf{H J}^{\mathbf{2}}$, \\ Shreenivas P. Revankar ${ }^{1 *}$
}

${ }^{1}$ Department of Pharmacology, Shimoga Institute of Medical Sciences, Shivamogga, Karnataka, India ${ }^{2}$ Department of Dermatology, Mc GANN Teaching Hospital, Shimoga Institute of Medical Sciences, Shivamogga, Karnataka, India

Received: 19 April 2016 Accepted: 03 June 2016

\section{*Correspondence to:}

Dr. Shreenivas P. Revankar, Email: sprevankar@yahoo.com

Copyright: () the author(s), publisher and licensee Medip Academy. This is an openaccess article distributed under the terms of the Creative Commons Attribution NonCommercial License, which permits unrestricted noncommercial use, distribution, and reproduction in any medium, provided the original work is properly cited.

\begin{abstract}
Background: Adverse drug reactions very often manifest in the form of cutaneous reactions. Majority of new drugs are developed by western countries and releases the drug worldwide after testing on small number of patients. Safety profile of such drugs may not be relevant in our country due to varied factors. Pharmacovigilance in the form of post-marketing surveillance helps to establish the accurate safety profile of drugs. The objective of this study was to study the various cutaneous adverse drug reactions (CADRs), frequency, type, severity and preventability of CADRs and to evaluate the drugs causing the CADRs.

Methods: A total of 52 patients were recruited for this study which was conducted in the dermatology OPD department from November 2015 to April 2016, demographic details, causality, severity, preventability were analysed by using standard scales.

Results: 52 patients with CADRs were included in the study during the 6 months study period. Results were presented in the form of number and percentage. Most common age group with CADRs was 40- 60 years; the most common suspected drug group causing CADRs was antimicrobials $46.15 \%$. According to Naranjos scale $67.30 \%$ of CADRs were probably caused by drugs. Schumoch and Thornton scale showed that $63.46 \%$ of CADRs in the study were definitely preventable.

Conclusions: Wide variety of drugs causes CADRs. Awareness among clinicians is required for active reporting of CADRs. Patients need to be educated for the cautious use of drugs causing ADRs to prevent the same.
\end{abstract}

Keywords: Post-marketing surveillance, CADRs, Naranjos probability scale

\section{INTRODUCTION}

At present large number of newer drugs are being introduced leading to 'drug explosion' and this has increased the chances of adverse drug reactions (ADRs). Many adverse effects of new drugs are only known during post marketing surveillance. The Ministry Of Health And Family Welfare, Government of India has set up national pharmacovigilance programme (NPP) to monitor adverse drug reactions (ADRs).
Pharmacovigilance plays a prominent role in establishing the safety profile of marketed drugs. ${ }^{1}$

Adverse drug reactions can cause decrease in the quality of life, increase physician visits, hospitalization and even death. ADRs can become a burden by causing changing over to other drugs, discontinuation of drugs and nonadherence to medications. ${ }^{2}$ CADRs are among the most frequent adverse drug reactions. ${ }^{3}$ 
Cutaneous adverse reactions are common but as many go un-reported, information regarding their incidence, severity and ultimate health effects are often not available. $^{4}$

In a study by Lihete et al the incidence of CADR's was high-7.02\% compared to other studies in which incidence of CADR's was much lower. ${ }^{5}$ In Abanti et al study the primary incidence of CADRs was $0.21 \%{ }^{6}$

Although the majority of CADRs are mild and selflimiting, severe manifestations such as toxic epidermal necrolysis (TEN) and Steven Johnson s Syndrome (SJS) are associated with a significant morbidity and might be fatal. ${ }^{7}$

Knowledge of drugs that can cause CADRs can help physicians in choosing safer drugs and therefore can be helpful to society at large. ${ }^{6}$ Health care professionals have a responsibility to their patients, who themselves are aware of the problems associated with drug therapy.

Since there are limited studies and information about occurrence and reporting of CADRs in clinical practice in hospitals of Karnataka the present study was done to assess the occurrence of adverse reactions and to identify the suspected drug causing the reaction in Mc.Gann teaching hospital a tertiary care hospital of shimogga.

\section{METHODS}

This was a prospective, descriptive, observational, noninterventional study conducted by department of pharmacology in collaboration with department of dermatology at district Mc.Gann teaching hospital Shivamogga Institute of Medical Sciences, Shimogga over a period of 6 months from November 2015 to April 2016. The study was approved by the institutional ethical committee of SIMS Shimogga.

All patients presenting to the dermatology out-patient department with suspected ADR s were enrolled in the study. Patients of all age group who presented with cutaneous lesions following intake of drugs were included. Patients with cutaneous lesions that were not due to drug intake and not willing to give informed consent were excluded from the study.

Demographic details like hospital number, age, sex, occupation, habits ,presenting complaints, duration of the illness history of drug allergy in the past, family history of drug allergy are noted. Types of reaction, nature of lesions, investigations if done were recorded with help of dermatologist.

Detailed drug history which included drug name (generic/branded), class, dose, frequency, duration, disease for which it was taken, Whether drug was prescribed or taken over the counter was also recorded in case record form. Treatment given for adverse drug reaction was recorded and whether the drug was discontinued, changed or dose was lowered was also recorded.

Probability assessment was done by Naranjos scale and reaction was graded as definite, probable, possible or doubtful. The preventability of adverse drug reaction was assessed by using the modified Schumock and Thornton scale as definitely preventable, probably preventable and not Preventable. Severity assessment was done by Hartwigs scale and reactions were classified as mild, moderate or severe.

All relevant data was recorded and analysed for the frequency of its occurrence and it was presented as percentage by using descriptive statistics.

\section{RESULTS}

The results are presented in the form of numbers and percentage, tables and figures were added for appropriate data to represent the results for easy understanding. The incidences of CADRs in the dermatology department were 52 patients out of 17,560 patients seen in 6 month study period $(0.29 \%)$. The most common age group in which CADRs was seen was 40-60 years (42.30\%), followed by $21-40$ years $(34.61 \%), 10-20$ years and beyond 60 years age group both had $6(11.5 \%)$ patients each. Of the patients with CADRs 25 were males $(48.07 \%)$ and $27(51.9 \%)$ were females. The major presenting complaints were rash $9(17.30 \%)$, rashes with intense itching 26 (50\%), itching 12 (23.07\%), hyperpigmentation $2(3.84 \%)$, eruptions with pain 2 (3.84\%). The most common suspected drug class causing ADR s were antimicrobials 24 cases $(46.15 \%)$, followed by NSAIDS 17 cases (32.69\%), antiepileptics 9 (17.30\%), steroids $2(3.84 \%)$ (Table 1$)$. The most commonly reported dermatological ADRs were urticaria $26(50 \%)$, followed by pruritis $12(23.07 \%)$, rashes $10(19.23 \%)$, hyper pigmentation $2(3.84 \%)$, fixed drug eruptions 2 $(3.84 \%)$. Among the 52 patients $10(19.23 \%)$ patients had type II diabetes, 5 (9.61\%) were hypertensive, 9 (17.30\%) had epilepsy as coexisting illness.

Table 1: Drugs causing CADRs.

\begin{tabular}{|ll|l|}
\hline Drug name & Frequency & Percentage \% \\
\hline Amoxicillin & 2 & 3.84 \\
\hline Ampicillin & 2 & 3.84 \\
\hline Azithromycin & 4 & 7.69 \\
\hline Cotrimoxazole & 6 & 11.5 \\
\hline Diclofenac & 9 & 17.30 \\
\hline Ibuprofen & 7 & 13.46 \\
\hline Paracetamol & 1 & 1.9 \\
\hline Phenytoin & 9 & 17.30 \\
\hline Steroids (topical) & 2 & 3.84 \\
\hline Quinolones & 10 & 19.23 \\
\hline Total & $\mathbf{5 2}$ & $\mathbf{1 0 0}$ \\
\hline
\end{tabular}


Table 1 showing drugs causing cutaneous adverse drug reactions in frequency and percentage based on drug name.

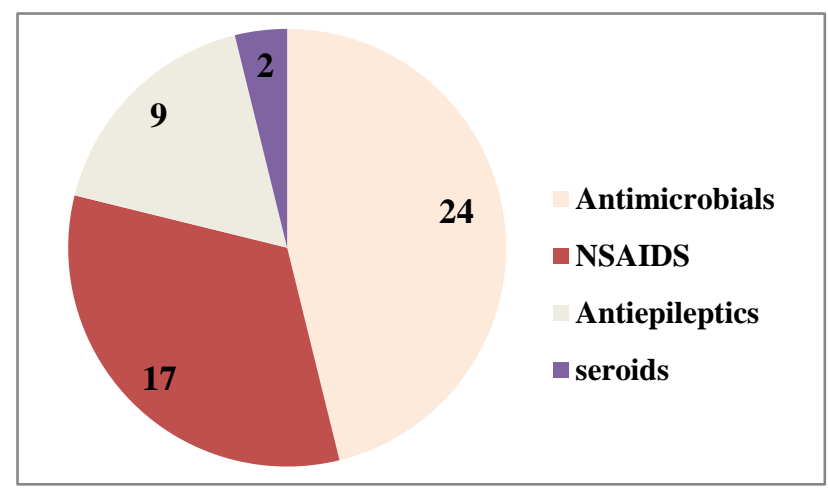

Figure 1: Drugs causing CADRS.

The measures taken against suspected drug were:

- Drug discontinuation, all 52 patients were discontinued from offending drug.

- Drug replaced, drug was replaced in 40 patients

- Dose reduction was not done in any patient

- Medications prescribed for management of ADRs was chlorpheniramine 4 cases, cetirizine 47 cases, and calamine lotion- 45 cases.

In the present study physician prescribed drugs causing ADRs were $40(76.9 \%)$ and 12 drugs were taken as over the counter medications $(23.07 \%)$ as self-prescribed medications. ADRs were grouped into 2 groups based on predictability of the reaction as type A, and type B, type A were 2 cases and type B were 50 cases.

Previous history of drug allergy was seen in 3 patients. Causative drug was not known. Family history of drug allergy was noted in 1 patient who had history of allergy in sibling but the causative drug was not known. Causality assessment was done by Naranjo s scale (Table 2).

Table 2: Naranjo's probability assessment.

\begin{tabular}{|lll|}
\hline Scale & Number of drugs & Percentage \% \\
\hline Probable & 35 & $67.30 \%$ \\
\hline Possible & 17 & $32.69 \%$ \\
\hline
\end{tabular}

Table 2 showing number and percentage of drugs causing CADRs, according to Naranjos probability assessment.

Table 3: Assessment according to Hartwigs severity scale.

\begin{tabular}{|lll|}
\hline Assessment & Frequency & Percentage \% \\
\hline Mild & 38 & $73.07 \%$ \\
\hline Moderate & 14 & $26.9 \%$ \\
\hline Total & $\mathbf{5 2}$ & $\mathbf{1 0 0}$ \\
\hline
\end{tabular}

Severity assessment was done by Hartwigs severity assessment scale (Table 3).

Table 3 showing frequency and percentage according to severity assessment by Hartwigs severity assessment scale.

\section{Table 4: Preventability assessment Schumoch and Thornton scale.}

\begin{tabular}{|lll|}
\hline Preventability & No. of CADRs & \% of CADRs \\
\hline Definitely preventable & 33 & $63.46 \%$ \\
\hline Probably preventable & 19 & $36.53 \%$ \\
\hline Not preventable & 0 & $0 \%$ \\
\hline Total & $\mathbf{5 2}$ & $\mathbf{1 0 0 \%}$ \\
\hline
\end{tabular}

Table 4 showing number of CADRs in number and percentage according to preventability assessment by Schumoch and Thornton scale preventability scale.

Preventability assessment was done by criteria by Schumoch and Thornton scale (Table 4).

\section{DISCUSSION}

Cutaneous reactions are the most common manifestation of ADRs. A wide range of cutaneous manifestations ranging from maculopapular rashes to Toxic epidermal necrolysis (TEN) can be produced by different classes of drugs. ${ }^{3}$ Fixed Drug eruptions are common, comprising $10-30 \%$ of all reported adverse drug reactions. ${ }^{9}$

The development of a cutaneous adverse drug reaction is frequently cited as a reason for discontinuation of treatment without completing therapeutic course. ${ }^{10}$

In this present study the incidence of CADRs in dermatology department was $0.29 \%$, this is low compared to other studies. In a study by Gohel et al shows that CADRs were $3.78 \%$ 2, some other studies shows incidence of dermatological ADRs in outdoor patients $2.3 \%$, Raut et al, $0.21 \%$ Padmavathi et al. ${ }^{4,11}$ The difference in incidence is due to difference in reporting and awareness about occurrence of CADRs. 14\% of ADRs are cutaneous or allergic in nature. ${ }^{12}$ In the present study a total of 52 CADRs were reported, the number does not represent the true incidence of CADRs during the period as many patients do not report minor CADRs or many CADRs are misdiagnosed as caused by other factors. In our present study, mild predominance of CADRs were seen in females (27 cases )compared to males 25 cases, which is similar to other studies, in a study by Nandha et al females had a higher predominance. ${ }^{3}$ Some studies showed that males had a higher predominance of CADRs, the difference may be that females report even minor CADRs and males usually ignore minor symptoms, Difference in pharmacokinetics, body weight and composition, hormonal effects on drug metabolism have been suggested as a possible explanation for the effect of gender on the ADEs. ${ }^{2,7}$ 
The most common age group in which CADRs was seen was $40-60$ years, 22 patients $(42.30 \%)$, followed by $21-40$ years, 18 patients $(34.61 \%), 10-20$ years and $>60$ years age group both had $6(11.5 \%)$ patients each, in a similar study by Sharma et al maximum number of cases were in the age group of $21-30$ years $(30.6 \%) .{ }^{13}$ In another study by Abanti et al majority of the patients were in the age group of $16-35$ years $(52.80 \%)$, varying age groups are due to different reporting rates of cases in different areas. ${ }^{4}$ In the present study out of 52 CADRs, 35 were probable $(67.30 \%)$ and 17 were possible $(32.69 \%)$, according to Naranjos causality assessment scale, a similar study showed probable cases as $85.10 \%$ and possible cases as $14.89 \%$ by Raut et al, no definite cases were seen in this study as drug was not re-administered and serum concentration was not measured, drug can be suspected to cause the ADR but definite confirmation that the reaction was caused due to the particular drug is not possible in every reported ADR as re-challenge with the suspected drug is risky and has ethical issues. ${ }^{11,14}$

Antimicrobial, antipyretic and anti-inflammatory agents remain the common culprits. ${ }^{15}$

All drugs are capable of producing any type of reaction in susceptible individuals but some drugs are more likely to induce certain reaction patterns and this can also give a clue regarding the likely causative drug. Anticonvulsants, anti-tuberculous drugs, pencillins, cephalosporins, sulfa group of drugs, Allopurinol and NSAIDs are more likely to produce Steven Jonhson syndrome/TEN spectrum of illness, FDE is commonly associated with NSAIDs, tetracyclins, doxycyclin, cotrimoxazole, pencillins, cephalosporins and phenobarbitone. ${ }^{16}$

In the present study suspected causative drugs were: antimicrobials 24 cases $(46.15 \%)$, followed by NSAIDS 17 cases (32.69\%), antiepileptics 9 cases (17.30\%), steroids 2 cases $(3.84 \%)$, among antimicrobials quinolones were $10(19.23 \%)$, cotrimoxazole $6(11.5 \%)$, azithromycin 4 (7.69\%), ampicillin 2 (3.84\%), amoxicillin $2(3.84 \%)$ among antiepileptics, phenytoin caused all the 9 cases $(17.30 \%)$, among NSAIDS, paracetamol caused 1 case $(1.9 \%)$, diclofenac caused 9 cases $(17.30 \%)$, ibuprofen caused 7 cases $(13.46 \%)$. In a similar study by Sharma et al causative drugs were antimicrobials in $40 \%$ followed by non-steroidal antiinflammatory drugs in $35.3 \%$, steroids $22 \%$, anticonvulsants $8 \% 13$, they had slightly lesser incidence caused due to antimicrobials $40 \%$, compared to the present study in which antimicrobials caused reactions in $46.15 \%$, there is slightly higher incidence due to NSAIDS $35.35 \%$, compared to the present study $32.69 \%$. In a study by Ghosh et al antibiotics caused reactions in $16(30 \%)$ cases, followed by anti-epileptics in 13 (25\%) cases, antitubercular drugs in $6(11 \%)$ cases, and antipyretics in $5(9 \%)$ cases. $^{8}$ In a study by Asawari et al the most common causative agents were antibiotics (48.9\%), NSAIDS (27.6\%), antiepileptic (10\%), antimicrobials $(04 \%)$, and antimalarials $(2.1 \%){ }^{11}$
No CADR with a new drug was observed in this study. One reason may be because of Government setup of this study and physicians usually prescribe from the hospital pharmacy, which sources drugs from essential drug list.

The most common reported dermatological ADRs were urticaria $26(50 \%)$, followed by pruritis $12(23.07 \%)$, rashes $9(17.3 \%)$, hyperpigmentation $2(3.84 \%)$, fixed drug eruptions $2(3.84 \%)$. In a study by Sharma et al Fixed drug eruption (FDE) was the most common CADR, seen 50 patients $(33.3 \%)$, followed by urticaria in 26 $(17.3 \%)$, maculopapular rash in $20(13.3 \%)$, acneiform eruptions in $17(11.3 \%)$, erythema multiforme (EM) in 15 $(10 \%) .{ }^{13}$ In a study by Asawari et al rashes on upper and lower limbs $(31.9 \%)$ was the most CADRs found in study followed by erythmatous and purpuric rash (14.8\%), maculopapular rash/eruptions $(12.7 \%)$, rashes all over body $(10.6 \%)$, erythmatous rash $(8.5 \%)$, urticaria $(8.5 \%)$ and Steven Johnnson syndrome $(4.2 \%){ }^{11}$ In a study by Saha et al among the various known patterns of CADR, morbilliformeruption (30.18\%), fixed drug eruption $(24.52 \%)$, and SJS-TEN and overlap of these two (24.50\%) comprised the bulk of the cases. ${ }^{6}$ In the present study physician prescribed drugs causing ADRs were 40 (76.9\%), 12 drugs were OTCs ( $23.07 \%)$, in a similar study by Saha et al incriminated drugs were mostly $(88.7 \%)$ physician prescribed. ${ }^{6}$ Lack of literacy and medical record keeping leads to repeated administration of drugs which increase the incidence and severity of ADR's which can be prevented by patient education and avoidance of self-administration. ${ }^{17}$

In the present study no patients had altered renal and liver function tests, peripheral eosinophilia was seen in 4 patients, eosinophil counts more than 1000 cells/cumm indicate a serious drug-induced cutaneous eruption. Thus, this can be a useful indicator among patients suffering from severe CADRs such as Steven Johnson syndrome, toxic epidermal necrolysis, and erythroderma. Altered liver and renal function tests predispose to severe CADRs because of abnormal drug metabolism and clearance from the body. ${ }^{13}$

In the present study according to Hartwigs severity assessment scale, mild reactions were $38(73.07 \%)$ and moderate reactions were $14(26.9 \%)$, in a similar study by Lihite et al, mild reactions were $78.76 \%$ and moderate reactions were $21.23 \%$, which had results slightly higher reactions in the group which were mild compared to the present study. ${ }^{5,14}$

In the present study, adverse events were classified as type A and type B according to Rawlins and Thompson classification, type-A (dose-dependent and predictable) or type-B (idiosyncratic, no clear dose response relationship), type A reactions were $2(3.8 \%)$, type $\mathrm{B}$ reactions were $50(96.15 \%)$ most of the reactions were not predictable and were in the type B category, in a similar study by Bhabhor et al type A-augmented 49 (44.14\%) and type B-bizarre category reactions were and 62 
$(55.86 \%){ }^{18,19}$ In another study by Padmavathi et al majority of the CADRs $38(92.6 \%)$ was of type B and the rest $3(7.4 \%)$ CADRs belonged to Type A. ${ }^{4}$

In the present study none of the reactions were due to herbal drugs this is because patients preferred modern medicine, in another study by Sudershan et al, herbal drugs caused $13.33 \%$ of cutaneous ADRs, Some herbal medicines in particular, ayurvedic remedies may contain arsenic or mercury that can produce typical skin reactions. Other popular remedies that can cause dermatological side effects include St. John's wort, kava, aloe vera, eucalyptus, camphor, henna and yohimbine. ${ }^{17}$

In the present study, according to Schumoch and Thornton preventability scale, 33 CADRs $(63.46 \%)$ were definitely preventable and $19(36.53 \%)$ were probably preventable, in a similar study by Padmavathi et al, definitely preventable were $12.2 \%$ and not preventable were $87.8 \%$, in another study by Inbaraj et al, all reactions were found to be preventable, the preventable reactions include reactions with previous allergic history to the drug or dose, frequency and route of administration inappropriate for the patient's condition, toxic serum concentration of the drug suggesting that inappropriately high dose was given or drug metabolizing enzymes were inhibited or suppressed, if there was a known treatment for the adverse drug reaction, the preventable causes should be detected as these drug reactions can be prevented and prevent distress and suffering to the patient. $4,18,20$

Limitations of this study were that only cases reported by patients were included in the study and unreported cases could not be documented, so the true incidence of CADRs could not be evaluated. Re-challenge with the suspected drug could not be done due to ethical reasons and unnecessary risk to the patients who might result in life threatening ADR. Serum concentration of the suspected drug was not monitored. Follow up of the patients was not possible and their final outcome after the suspected CADR could not be recorded.

\section{CONCLUSION}

Wide variety of drugs cause CADRs .Awareness among clinicians is required for active reporting of CADRs .The CADRs reported were varied, the drugs causing them were commonly prescribed drugs in this study. Awareness about CADRs is needed for pharmacovigilance activities and even minor non-serious reactions must be reported to obtain the actual occurrence of CADRs .Drugs which cause CADRs must be documented and repeat administration of the same drug in the patient should be completely avoided, if the drug has caused a serious reaction.

\section{AKNOWLEDGEMENTS}

The Authors of this study are highly thankful to the staff of the Department of Dermatology, Mc.Gann teaching hospital, SIMS, Shivamogga for their help and cooperation in conducting the present study.

Funding: No funding sources

Conflict of interest: None declared

Ethical approval: The study was approved by the Institutional Ethics Committee SIMS/IEC/239/2015-16

\section{REFERENCES}

1. Srivastava SK. A complete textbook of medical pharmacology, $1^{\text {st }}$ edition, Avichal publishing company. 1:81-91.

2. Gohel D, Kumar BS, Malhotra S. Evaluation of dermatological adverse drug reaction in the outpatient department of dermatology at a tertiary care hospital. Indian Journal of Pharmacy Practice. 2014;7(3)42-9.

3. Nandha R, Gupta A, Hashmi A. Cutaneous adverse drug reactions in a tertiary care teaching hospital: a North Indian perspective. International Journal of Applied and Basic Medical Research. 2011;1(1):503.

4. Padmavathi S, Manimekalai K, Ambujam S. Causality, severity and preventability assessment of adverse cutaneous drug reaction. A prospective observational study in a tertiary care hospital. Journal of Clinical and Diagnostic Research. 2013;7(12):2765-7.

5. Lihite RJ, Lahkar M. A study on cutaneous adverse drug reactions in adr monitoring centre of tertiary care hospital, Guwahati. J App Pharm Sci. 2013;3(3):79-81.

6. Abanti S, Kanti DN, Avijit H, Chandra GR, Nath CS, Kanti DP. Cutaneous adverse drug reaction profile in a tertiary care out-patient setting in Eastern India. Indian Journal of Pharmacology. 2012;44(6):792-7.

7. Mokhtari F, Nikyar Z, Naeini BA, Esfahani AA, Rahmani S. Adverse cutaneous drug reactions. Eight year assessment in hospitalized patients. J Res Med Sci. 2014; 19:720-5.

8. Ghosh S, Acharya LD, Rao GM. Study and evaluation of the various cutaneous adverse drug reactions in kasturba hospital, Manipal. Indian journal of pharmaceutical sciences. 2006;68(2):2125.

9. Ding WY, Lee CK, Choon SE. Cutaneous adverse drug reactions seen in a tertiary hospital in Johor, Malaysia. International Journal of Dermatology. 2010;49:834-41.

10. Lamani VL, Ratnakar JS, Kotinatot BC, Bhushan A. Study of cutaneous adverse drug reactions in a tertiary care teaching hospital. International Journal of Basic and Applied Medical Sciences. 2015;5(1):71-4. 
11. Raut A, Pawar A, Pankaj M, Srivastava P, Mishra A. Clinical pattern and severity of cutaneous adverse drug reactions. International Journal of Pharmacy and Pharmaceutical Sciences. 2013;5(2):612-6.

12. Shear NH. Cutaneous reactions to drugs. In: Freedberg IM, Eisen AZ, Wolff K, Austen KF, Goldsmith LA, Katz SL. Fitzpatrick's dermatology in general medicine. Sixth edition, McGraw-Hill; 2003:I:1330-133.

13. Sharma R, Dogra D, Dogra N. A study of cutaneous adverse drug reactions at a tertiary center in Jammu, India. Indian Dermatology Online Journal. 2015;6(3):168-71.

14. Srinivasan R, Ramya G. Adverse drug reactioncausality assessment. International Journal of Research in Pharmacy and Chemistry. 2011;1(3):606-12.

15. Patel NH, Padhiyar J, Shah YB, Dixit RK. Study of causality, preventability and severity of cutaneous adverse drug reactions in a tertiary care institute. GCSMC J Med Sci. 2015;4(1):24-7.

16. Sasidharanpillai S, Riyaz N, Khader A, Rajan U, Binitha MP, Sureshan DN. Severe cutaneous adverse drug reactions. A clinicoepidemiological study. Indian J Dermatol. 2015;60:102.

17. Sudershan V, Siddiqua S, Aruna D, Manmohan, Ramesh S, Yasmeen N. Cutaneous adverse drug reactions in a tertiary care hospital . Der Pharmacia Lettre, 2011;3(6):210-7.

18. Raut AL, Patel P, Patel C, Pawar A. Preventability, predictability and seriousness of adverse drug reactions amongst medicine inpatients in a teaching hospital. A prospective observational study. International Journal of Pharmaceutical and Chemical Sciences. 2012;1(3):1293-9.

19. Bhabhor PH, Patel TK, Vahora R, Patel PB, Desai N. Adverse drug reactions in a tertiary care teaching hospital in India: analysis of spontaneously reported cases. Int J Basic Clin Pharmacol. 2014;3:1078-85.

20. Inbaraj SD, Muniappan M, Muthiah NS, Arul A, Glory JI, Farhana R. Pharmacovigilance of the cutaneous drug reactions in outpatients of dermatology department at a tertiary care hospital. Journal of Clinical and Diagnostic Research. 2012;6(10):1688-91.

Cite this article as: Murray JL, Vedavathi $\mathrm{H}$, Prasad NS, Dadapeer HJ, Revankar SP. A study on cutaneous adverse drug reactions at district Mc. Gann teaching hospital, Shimoga institute of medical sciences, Shivamogga, Karnataka, India. Int J Basic Clin Pharmacol 2016;5:1343-8. 